\title{
Clinical Value of Color Doppler Ultrasound in Diagnosis of Children Intussusception
}

\author{
Li Kaiwen \\ Department of Ultrasound, Jingzhou Central Hospital, The Second Clinical Medical College, Yangtze University, Jingzhou, China
}

Email address:

likaiwen1234@163.com

To cite this article:

Li Kaiwen. Clinical Value of Color Doppler Ultrasound in Diagnosis of Children Intussusception. International Journal of Medical Imaging. Vol. 6, No. 1, 2018, pp. 9-11. doi: 10.11648/j.ijmi.20180601.12

Received: February 6, 2018; Accepted: February 25, 2018; Published: March 20, 2018

\begin{abstract}
To analyze and evaluate the characteristics and effects of ultrasonography in the diagnosis of intussusception in children, the clinical data of 65 children with intussusception diagnosed by ultrasound were retrospectively analyzed. As a result, this group of children with intussusception confirmed by X-ray air enema reduction or surgery were diagnosed with no false-positive cases by color Doppler ultrasound. The characteristics of intussusception in children were as follows: the cross section of condom was changed concentrically, showing a "target ring" sign, a ring-shaped strong echo was found in the center of the target ring, or gas-liquid mixed echo. The outer circumference of the target ring alternately surrounds the multiple layers of strong and weak echoes. The thickness of the target ring is closely related to the number of layers of the intestine and the edema of the intestinal wall. The longitudinal section of the nested pile shows sleeve-like changes, showing a "sleeve" sign, the length in the range of 3.6 to $6.2 \mathrm{~cm}$. Finally, with reference to the characteristic changes of ultrasonic images in pediatric intussusception, it is concluded that the diagnosis of intussusception in children is reliable and accurate, and it is worthy of wide clinical application.
\end{abstract}

Keywords: Children Intussusception, Diagnosis, Low Frequency Ultrasound, High Frequency Ultrasound, Clinical Value

\section{Introduction}

Intussusception is formed by a part of the intestine and its attached mesentery into the adjacent intestinal cavity. Intussusception in children, also known as idiopathic intussusception, accounts for about $95 \%$ of all children's intussusception, which is one of the most common acute abdomen in the clinic of children. If the treatment is not in time, it will lead to serious consequences. Timely and correct diagnosis and treatment can avoid intestinal necrosis and reduce unnecessary pain. To analyze and evaluate the characteristics and effects of ultrasonography in the diagnosis of intussusception in children, the clinical data diagnosed by ultrasound were necessary and meaningful. Apparently, for the advantages of no trauma and no pain of ultrasonic diagnosis, it is an ideal method for the examination of children's disease in the present. The advantages of ultrasonic diagnosis of intussusception in children are mainly manifested by the easy acceptance of children and the diagnostic accuracy of this method is high [1]. From January 2016 to July 2017, 65 cases of infantile intussusception were diagnosed by ultrasound and confirmed by operation or X-ray air enema reduction. The diagnosis is reported as follows:

\section{Data and Methods}

\subsection{General Information}

From January 2016 to July 2017, 65 cases of infantile intussusception were diagnosed by ultrasound and confirmed by operation or X-ray air enema reduction, including 39 males and 26 females; children aged 3 months $\sim 5$ years old, an average of 1.7 years old; onset of illness in children were between $5 \sim 60 \mathrm{~h}$. Clinical manifestations included 57 children with crying, bloating, abdominal pain and vomiting, 52 with blood in the stool, 59 with palpable mass in the abdomen, and 31 with fever.

\subsection{Equipment and Testing Methods}

Philips iU22 and Mindray DC-8 color Doppler ultrasound diagnostic system is used for diagnosis. At the time of detection, the abdominal low frequency probe is set up, and the frequency is set to $3.5-5.0 \mathrm{MHz}$. Then the high frequency probe is used, and the frequency is set to $7.5-10.0 \mathrm{MHz}$, then 
children are examined in turn. Help the child to take a sleeping position, and keep it quiet under the help of the nursing staff and family members. First, use the abdominal low frequency probe to detect the abdomen, determine whether there is mass or swelling in the abdomen, and assess whether the intestinal peristalsis is normal, and whether there is fluid in the abdominal cavity. Then use high-frequency probe, the suspicious masse for a second inspection for the main observation of the internal structure of mass, to determine its nature. The CDFI (color Doppler flow imaging) was then used to conduct a comprehensive examination of intestinal blood flow signals in the intussusception area.

\section{Results}

This group of children with intussusception confirmed by $\mathrm{X}$-ray air enema reduction or surgery were diagnosed with no false-positive cases by color Doppler ultrasound. A total of 42 children had a small amount of ascites. The characteristics of intussusception in children were: concentric changes in the cross-section of the nested area, showing "target ring" sign (see Figure 1), circular strong echoes found in the center of the target ring, or gas Liquid mixed echo, the outer ring of the target ring alternates with multiple layers of strong and weak echoes alternately, and its thickness is closely related to the number of layers inserted into the midgut and the edema of the intestinal wall. The longitudinal section of the nested sleeve shows a sleeve-like Change, showing a "sleeve" sign, the length in the range of 3.6 to $6.2 \mathrm{~cm}$.

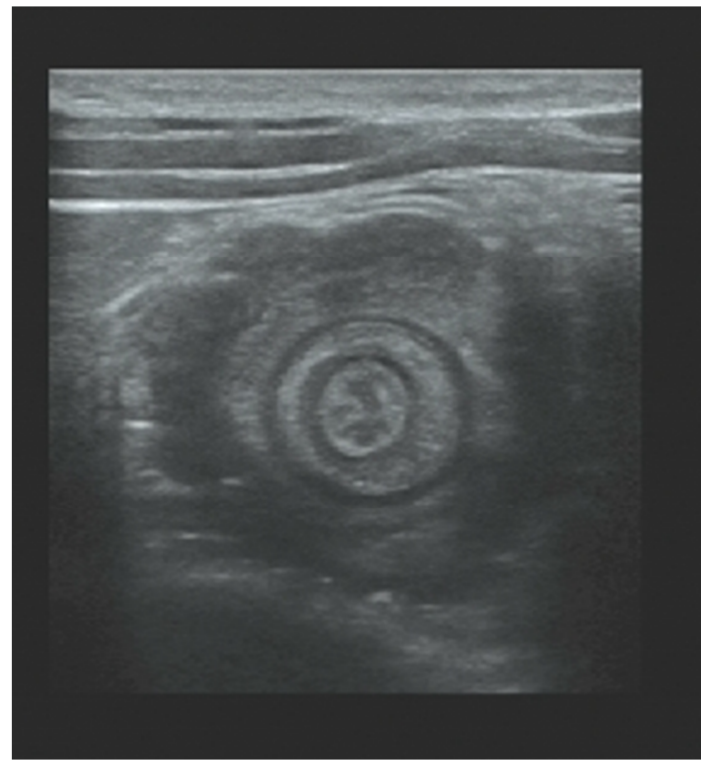

Figure 1. "target ring" sign of intussusception.

\section{Discussion}

Intussusception refers to the intestinal canal and the mesentery inserted into the adjacent intestinal cavity. In short, the proximal cavities are inserted into the internal [2] of the distal lumen. The causes of intussusception in children include diet change, infection and diarrhea. The symptoms are paroxysmal abdominal pain, vomiting, crying, jam-like feces, etc. a few children can feel abdominal sausage like-lumps. The age of the children is small, the coordination of the treatment is poor, and the early diagnosis of the disease is more difficult, so the early diagnosis is not good [3]. High frequency ultrasound has a high resolution, which can clearly reflect the shape of the intussusception. After transversely scavenging, the image of the concentric circle was presented, and the outer circle was low echo and even, which is the echo [4] of the distal intestinal canal wall. The strong echo ring may be the mesenteric fat, the oedema serosa or the gas between the sheath and the interset. Hypoechoic is the edema of the intestinal wall, and the anechoic or low echo in the central position should be the exudate of the edema or the [5] of the mucous membrane. If the mesenteric knot is inserted, the lymph node hypoechoic group can be displayed in the central part. The longitudinal section was characterized by sleeve, showing 4 parallel strong echoes, and a strong echo in the outer layer, which is the edema and serosa and adjacent interface of the intussusception sheath. The wider internal hypoechoic band is the formation of sheath and edema of intestinal wall in inserted parts [6]. The middle part is hypoechoic and is arranged in parallel with the multilayers of intestines. The internal strong echoes are caused by the residual gas, mesangial fat and intestinal contents in the intestinal cavity. With the support of the above imaging features, the superimposed CDFI can observe the mesenteric blood flow signal (see Figure 2), which is an effective way to determine the blood circulation in the intestinal tract, and [7] can be formulated in turn. If the intussusception mass does not have blood flow signals, and there is a free fluid echo in the abdominal cavity, and the edema phenomenon of the intestinal wall is more obvious, it may be intestinal necrosis, requiring air enema treatment or surgical treatment. The data show that after a comprehensive diagnosis of infantile intussusception, the earlier the enema is, the higher the cure rate is [8].

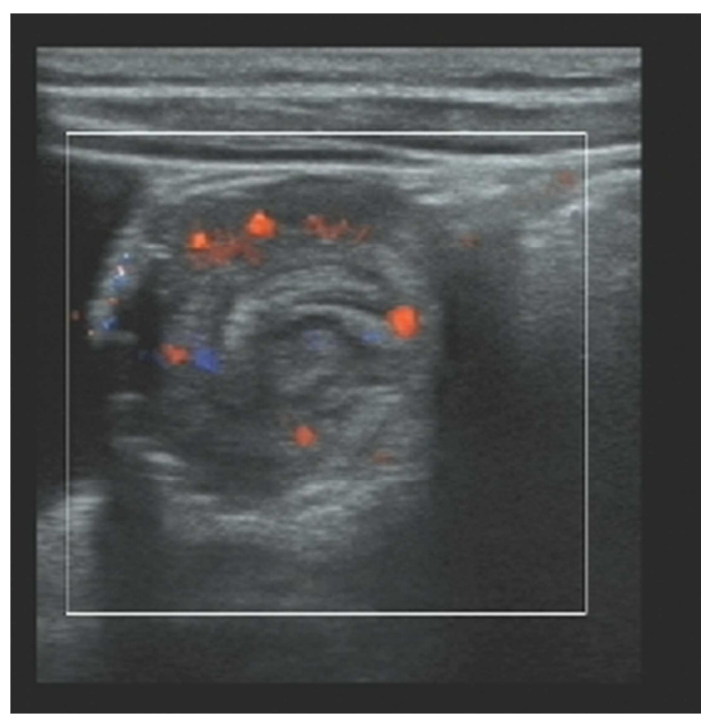

Figure 2. CDFI in the intestinal tube at the intussusception.

In this study, 59 of the 65 cases of intussusception were 
typical "concentric circle sign" and "sleeve sign", accounting for $90.8 \%$. Intussusception in children is usually single, but there are also 2 or more cases of intussusception. In this study, 1 case of children had intussusception at the right upper abdomen and left lower abdomen. Therefore, when ultrasonic scanning was performed, you could not find a 1 lesion to give up the examination of other parts, otherwise misdiagnosis would be occurred. Ultrasonic diagnosis of intussusception should be distinct from the emptying of the gastric antrum and appendicitis. The gastric emptying may be transient performance as "concentric circle sign", but would disappear with the dynamic observation of intestinal peristalsis. The appendicitis diagnosis standard is appendicitis thickening, wall circumference and appendiceal cavity effusion, its cross section can also be "concentric circle" shape, but its concentric circle diameter is often smaller than intussusception, and the location of onset is mostly in Maer area. A total of 60 intussusception masses were found in this group of cases, and 47 in the right upper abdomen, accounting for $78.3 \%$. Acute infantile intussusception is an acute abdomen. The key to the treatment is early detection and early diagnosis. The sonographic features of intussusception complicated by intestinal necrosis: (1) intestinal wall thickening, hypoechoic, peristalsis weaken or disappear, overlapping of effusion; (2) a typical expression of peritoneal effusion is intussusceptional necrotic bowel; (3) color Doppler ultrasound to detect blood flow signal. Within a block or only of scattered blood flow signal, indicating intestinal necrosis may be [9-11]. In this study, 11 cases of children who underwent surgical treatment at the end of the operation were all over the time of $48 \mathrm{~h}$. There were different degrees of intestinal necrosis during operation. The longer the onset time is, the worse the prognosis is. Of course, there are limitations in ultrasound diagnosis of intussusception. When diagnosing the location of intussusception, it can only be roughly judged according to the location of the lesion. It is difficult to distinguish the specific parts of jejunal intussusception or ileum intussusception. However, high frequency ultrasound has a high accuracy in the diagnosis of intussusception in children, especially color Doppler ultrasound, which can provide an important basis for clinicians to choose treatment plan.

\section{Conclusion}

In summary, to grasp the intussusception "concentric", "sleeve sign" these two characteristic ultrasound manifestations, it can be diagnosed quickly and accurately when combined with clinical history and a careful and comprehensive scan. These two characteristic ultrasound manifestations provide a basis for clinical diagnosis. They are of great practical value in the clinical diagnosis and treatment of intussusception in children and can be used as the first choice for the diagnosis of intussusception in children.

\section{References}

[1] Xuan Aijun, Yang Guoqiang. Pediatric intussusception ultrasound diagnosis [J]. Chinese Journal of Ultrasound Diagnostics, 2003, 4 (8): 603-604.

[2] Wang Yumin, Honghua, Wang Fang, and so on. Color Doppler ultrasound diagnosis of pediatric intussusception [J]. Chinese Journal of Health Nutrition, 2016, 26 (14): 219-220.

[3] Chen Fu Fu. Color Doppler ultrasound diagnosis of pediatric intussusception [J]. Medical Frontier, 2014, 8 (27): 137.

[4] Yao Wei right. Value of color Doppler ultrasound in the diagnosis of pediatric intussusception [J]. Modern Medical Imaging, 2016, 25 (4): 764-766.

[5] Yu Yong, He Chun, Li Yingqing, and so on. Value of color Doppler ultrasound diagnosis of 162 cases of children intussusception value [J]. Modern Medical Imaging, 2015, 24 (5): 772 .

[6] Wang Yingqi, He Xiaoping, Hu Chunmei, et al. Color Doppler ultrasound diagnosis of intussusception in children and ultrasound-guided reduction of water enema clinical value $[\mathrm{J}]$. Chinese Medical Equipment, 20 15, 9 (3): 73-75.

[7] Lu Shouyin, Luo Aeqin, Zhou Pigeon, and so on. Clinical value of high frequency ultrasound diagnosis of intussusception in children [J]. Journal of Practical Medicine, 2015, 32 (1): 19-20.

[8] Liu Pingping, Tian Xiaoxian. High-frequency color Doppler ultrasound in children with primary and secondary intussusception value [J]. Medical Information, 2014, 6 (14): 180.

[9] Lou Zhifeng, Teng Xiang. Discussion of the association of mesenteric lymphadenitis with intussusception in children $[\mathrm{J}]$. Chinese Practical Medicine, 2016, 6:20-22.

[10] Chen Haiyan, Liu Jiangze, Hu Xiaowei, et al. Color Doppler ultrasound in diagnosis and guidance of water enema reduction for intussusception in children: [J]. medical information, 2013, 26 (3): 84-86.

[11] Kou Guangling, Huang Jin, Liu Diantao, et al. Application of color Doppler ultrasonography in diagnosis of intussusception in children and ultrasound monitoring of saline enema reduction $[\mathrm{J}]$. Chinese Journal of ultrasound medicine, 2014, 30 (12): 1111-1113. 